\title{
Nuclear Medicine Physics: Review of Advanced Technology
}

\author{
Jungsu S. Oh ${ }^{(1)}$ \\ Department of Nuclear Medicine, Asan Medical Center, University of Ulsan College of Medicine, Seoul, Korea
}

Received 29 May 2020

Revised 29 July 2020

Accepted 19 August 2020

Corresponding author

Jungsu S. Oh

(jungsu_oh@amc.seoul.kr)

Tel: 82-2-3010-4598

Fax: 82-2-3010-4588
This review aims to provide a brief, comprehensive overview of advanced technologies of nuclear medicine physics, with a focus on recent developments from both hardware and software perspectives. Developments in image acquisition/reconstruction, especially the time-of-flight and point spread function, have potential advantages in the image signal-to-noise ratio and spatial resolution. Modern detector materials and devices (including lutetium oxyorthosilicate, cadmium zinc tellurium, and silicon photomultiplier) as well as modern nuclear medicine imaging systems (including positron emission tomography [PET]/computerized tomography [CT], whole-body PET, $\mathrm{PET} /$ magnetic resonance [MR], and digital PET) enable not only high-quality digital image acquisition, but also subsequent image processing, including image reconstruction and postreconstruction methods. Moreover, theranostics in nuclear medicine extend the usefulness of nuclear medicine physics far more than quantitative image-based diagnosis, playing a key role in personalized/precision medicine by raising the importance of internal radiation dosimetry in nuclear medicine. Now that deep-learning-based image processing can be incorporated in nuclear medicine image acquisition/processing, the aforementioned fields of nuclear medicine physics face the new era of Industry 4.0. Ongoing technological developments in nuclear medicine physics are leading to enhanced image quality and decreased radiation exposure as well as quantitative and personalized healthcare.

Keywords: Nuclear medicine physics, Advanced techniques, Theranostics, Precision medicine, Deep learning, Personalized healthcare

\section{Introduction}

\section{Overview of nuclear medicine physics}

Nuclear medicine physics (NMP) encompasses many research areas, including atomic/nuclear physics (e.g., various radioactivity decay modes), hardware-related fields (e.g., radiation detectors and related electrical instruments), counting statistics/systems, software-related fields (e.g., digital image processing), tracer kinetic modeling, and internal/external radiation dosimetry [1]. Due to the large coverage with limited space and time for the 30th anniversary Progress in Medical Physics (PMP) review series, this review focuses on recent advances of NMP for in vivo NM imaging. Before leaping from the discovery of radioactivity to modern NMP, it is worth summarizing the history of NM and NMP.

\section{History of NM and NMP}

Radioactivity was discovered by Henri Becquerel in 1896 (one year after the discovery of the X-ray by Roentgen), 
Jungsu S. Oh : Nuclear Medicine Physics: Review of Advanced Technology

and shortly thereafter, Marie Curie (mother of Irene Curie) coined the term radioactivity and developed the theory of radioactivity; both were awarded the Nobel Prize in Physics in 1903 [2]. Thereafter, there have been dramatic achievements in many aspects of NMP.

Artificial radioactivity was invented by Irene and Joliot Curie in 1934, and in the meantime, Ernest Lawrence invented the cyclotron (i.e., device for accelerating atoms at high speed to produce radioisotopes) in 1930 and continued to develop the medical cyclotron. These two inventions led to the production of radionuclide for medical use (e.g., I-131 and Tc-99m) [2,3].

Ernest Rutherford identified alpha and beta rays and proposed the nuclear theory of the atom, which is the basis of not only NM therapeutics, but also recent cutting-edge technology of theranostics, and was awarded Nobel Prizes in Chemistry in 1908 and 1919 [4].

NM first gained public recognition through radioactive iodine (RAI) research by Hertz and Roberts [3,5]. For NM imaging, Hans Geiger invented the first practical scintillation detector called the Geiger counter using the GeigerMuller tube in 1928 [6], which is the basis of modern radiation detection systems and led to the first NM imaging study for mapping thyroid iodine uptake [7]. Cassen et al. [8] developed the first rectilinear scanner with a motor-controlled/collimated radiation detector, and printer, founding the basis of single-photon emission computed tomography (SPECT) and coincidence detection of positron emission tomography (PET) $[3,9,10]$. The first gamma camera was the scintillation camera developed by Hal Anger [10].

Paul Dirac discovered the existence of annihilation positrons and received the Nobel Prize in Physics in 1933 [3] (PET is based on the coincidence of an opposite-direction 511-keV annihilation photon pair originating from this phenomenon). More recently, PET was invented by Kuhl and Edwards [11] in the early 1950s, who performed a preliminary version of reconstruction followed by coincidence imaging without lead collimation [12]. In the meantime, the concept of emission/transmission tomography was also developed into SPECT [3].

\section{Two major branches of NM imaging}

Unlike other structural imaging, such as X-ray computerized tomography (CT) or ultrasound, traditional NM imaging (i.e., gamma camera/SPECT) and PET provide images reflecting physiological functions. To this end, both PET and SPECT require a radiopharmaceutical to be distributed into the whole body or organs-of-interest of the subject depending on the purpose of the imaging test.

Although the author will discuss NMP in both modalities, modern NM imaging is dominated more by PET, due in particular to the high image quality in terms of the signal-tonoise ratio (SNR)/spatial resolution. Therefore, the author will focus more on NMP advances of PET. Broadly speaking, most innovative physical methods are first developed for PET imaging and are then incorporated into traditional NM imaging.

\section{NM imaging as picomolar-sensitive molecular imaging}

Before addressing NMP advances in image acquisition/ reconstruction and related hardware/software developments, an overview is provided of PET, which is a major component of modern NM/NMP.

As summarized by Jones [13], PET is a quantitative imaging modality with picomolar sensitivity. Whereas other imaging modalities focus mostly on structural imaging of the human body, NM imaging (PET in particular) focuses on physiology, metabolism, drug distribution, and molecular pathways/targets. At present, PET is widely considered the most sensitive technique available not only for noninvasively studying how the human body functions in terms of physiology, metabolism, and molecular pathways, but also for studying which in vivo pathologies (e.g., amyloid and tau for Alzheimer's disease) are in the human body. In this regard, PET scans sometimes lead other modalities in terms of disease detection and early diagnosis. PET tracers should thus be developed to address clinical research, diagnostic questions, and challenges to properly distribute radiopharmaceuticals in the body (mostly by injection, but sometimes by swallowing and inhalation for organs/tissues of interest). 
As mentioned, gamma camera (or SPECT) imaging is another major NM imaging modality. Because most gamma tracers can be easily synthesized using a generator-based radioisotope (mostly for Tc-99m), there are many useful NM imaging tests using this modality. Nonetheless, because many technological aspects are led by PET, the author focuses first on recent advances in PET image acquisition/ reconstruction/processing.

\section{Limitations and solutions for NM/PET imaging}

Although PET is one of the most molecular-sensitive imaging techniques and has significantly better sensitivity and spatial resolution than gamma camera/SPECT owing to collimation-free three-dimensional (3D) imaging, the counting statistics are still intermediate in that PET is a photondeficient modality compared with other modalities, such as X-ray CT. Therefore, PET has a low SNR and potential limitations in reducing the imaging time and radiation dose. In addition, there are well-known fundamental limitations of PET spatial resolution [14] compared with other structural imaging, such as X-ray CT and magnetic resonance (MR) imaging due to many factors, including the physical size of the detector elements, non-collinearity of the line of response (LOR), and positron range.

To resolve these limitations in PET and PET/CT imaging, NMP has evolved greatly over the last two decades thanks to hardware developments of hybrid imaging [15] free from PET transmission imaging, faster detector systems [16-20], and corresponding software developments [21]. The present standard of PET/CT scanners has incorporated many advanced hardware/software methods in recent decades. First, benefits are provided by the flexible structures of iterative algorithms, including ordered subset expectationmaximization (OSEM) and maximum a posteriori (MAP) algorithms, which have made it possible to achieve a tradeoff between SNR and spatial resolution and to further consider noise penalization, respectively. Second, there have been methods for increasing the effective sensitivity of the PET camera, including the time-of-flight (TOF) technique and whole-body PET. Third, there have been methods for improving the spatial resolution of the PET camera: the point-spread function (PSF), depth-of-interaction (DOI), and partial volume correction (PVC) techniques.

These developments in NMP have led to better image quality in terms of both sensitivity and spatial resolution, and have thus improved small lesion detectability (in tumor imaging, including FDG PET) [22], in vivo pathology detectability (in neurodegenerative disease imaging, including amyloid and tau PET) [23,24], and the general quantification accuracy of PET uptake.

\section{Standardized uptake value metric}

The Standardized uptake value (SUV) is a well-established (semi-)quantitative measure for PET, playing an especially large role in whole-body oncology PET studies. The SUV is a ratio between concentrations and can be calculated by the activity concentration in a tumor $([\mathrm{Bq} / \mathrm{mL}] /$ injected activity $[\mathrm{Bq}] \times$ body weight $[\mathrm{g}]$; $\mathrm{mL}$ : milliliter, Bq: Becquerel). Maximum uptake in a tumor (i.e., SUVmax) is one of the most frequently used metrics not only because it can be easily measured, but also because its diagnostic accuracy is the highest among many FDG PET metrics. Recently, the metabolic tumor volume (MTV) and total metabolic burden (MTV×SUVmean) within the volume called total lesion glycolysis are considered additional useful metrics.

Because SUVmax is sensitive to not only to the PET camera characteristics, but also noise and reconstruction parameters, 1-mL spherical volume-of-interest (VOI)-based maximum uptake is also used and referred to as SUVpeak. PERCIST criteria adopt this as the metric of choice and highly recommend it for tumor assessments between subjects/centers and baseline/follow-up studies [25].

\section{Scope of this review}

This review offers a brief, comprehensive overview of advanced technologies of NMP to provide knowledge to medical physicists, NM physicians, and researchers. Because NMP has both hardware and software aspects, the author focuses on recent developments/advances in both aspects. Developments in image reconstruction, especially TOF and PSF, have potential advantages in terms of image SNR and spatial resolution. Regarding hardware systems, 
modern detector materials and devices, including lutetium oxyorthosilicate (LSO), the silicon photomultiplier (SiPM), and NM imaging systems (PET/CT, PET/MR, digital PET, whole-body PET), will be addressed.

NMP has distinctive characteristics of molecular imaging and multidisciplinary and multicenter study-related physics (in particular, harmonization methods) compared with other MP subspecialties. In this regard, the review also includes current perspectives of cutting-edge NM techniques (e.g., theranostics and deep learning [DL] in NM). For the review specific to the Korean NMP society, the author will discuss future challenges and roles of the Korean NMP society as benchmarking with other NMP societies, and will suggest corresponding perspectives for the Korean NMP society in the Discussion and Perspective section.

\section{NMP Advances in Image Acquisition/Reconstruction}

\section{Technique for increasing SNR}

\section{1) TOF PET}

Whereas a conventional PET camera uses coincidence information only for determining the LOR of both oppositedirection annihilation gamma rays, TOF PET resolves the difference in arrival time between both gamma rays to determine the approximate annihilation position on the LOR. By reducing the uncertainty of the annihilation position, TOF PET can improve the effective sensitivity and SNR, which is proportional to the square root of noise equivalent count (NEC) [26], where NEC is reciprocal to the uncertainty. Moreover, TOF PET requires neither massive modification of the PET detector geometry nor other image degradation (e.g., spatial resolution). Rather, the increased SNR permits improved trade-off between SNR and spatial resolution. Therefore, TOF PET has become the standard PET imaging technique.

Although the principle of TOF was proposed earlier, its practical implementation was delayed by several decades [27]. Only prototypes during the 1980s [28-34] used BaF2 or $\mathrm{CsF}$ as scintillation crystals for the purpose of reducing decay time compared with high-density scintillation crystals (i.e., bismuth germinate or bismuth germanium oxide
[BGO]-based systems). However, these scintillation crystals have limited stopping power and light output; thus, these systems suffered from poor sensitivity and spatial resolution. Therefore, a BGO-based PET camera was the method of choice during that period, and only limited clinical studies and research-based experiments were conducted on TOF PET cameras [28].

LSO became available in the 1990s, thus making it possible to implement practical TOF PET, which requires not only high timing resolution, but also adequate stopping power and energy resolution. The combination of faster detector materials (e.g., LSO or lutetium-yttrium oxyorthosilicate [LYSO]), faster photomultiplier/electrics (i.e., with high temporal resolution), and faster computing power [35] was a critical prerequisite for achieving the clinically useful TOF technique. In addition, list-mode-based iterative reconstruction algorithms played an important role for TOF PET with the assistance of an iterative approach, which has a flexible pipeline in its iterative nature and can thus modify the algorithm to incorporate TOF information.

Due to the patent by Siemens, a similar variation of the lutetium-based scintillation crystal (i.e., LYSO) was introduced in the early 2000s by other vendors. Taken together, TOF PET became the method of choice in the 2000s [16-20], providing a timing resolution of $350-550 \mathrm{ps}$ and a localization uncertainty of 5-8 $\mathrm{cm}$. With improvements in the related electrics, the uncertainly is decreasing daily ( $200 \mathrm{ps}$ at present).

\section{2) Whole-body PET}

Whole-body PET is the epitome of increased sensitivity in PET. The world's first whole-body PET/CT scanner was developed by Cherry et al. [36]. By increasing the geometric coverage (known as the solid angle in NMP) to encompass the entire body, the sensitivity could be increased by a factor of approximately 40 for whole-body imaging. Moreover, significant advances in the TOF technique arising from ongoing improvements in timing resolution may have contributed to achieve improved effective sensitivity. The overall sensitivity gains are considered to be more than hundreds of times greater than current state-of-the-art (i.e., bedposition-based) PET cameras.

Cherry et al. [36] also demonstrated how this step change 
in sensitivity with unprecedented count density affects the way PET is used both in clinical research and patient care. His colleagues also reported the first-in-human imaging study using the 194-cm axial field-of-view (FOV) wholebody PET/CT scanner called the EXPLORER, obtaining the total adult body in a single acquisition, confirming wholebody pharmacokinetic studies with 1-s frame durations [37]. Moreover, they suggested that large gains in sensitivity and SNR can be alternatively used for low-dose (25 MBq or less within the FOV) or short-scan-duration ( 1 min or less) PET imaging.

\section{Techniques for improving spatial resolution}

\section{1) PSF iterative reconstruction and DOI technique}

PSF-based iterative reconstruction uses a system matrix to couple coincidence counts along each LOR to activities in neighboring voxels to account for all processes affecting the LOR, including the positron range, photon non-collinearity, and detector geometry-based effects, such as intercrystal scattering/penetration $[22,38]$. In this regard, the central LOR with less crystal penetration and the off-central LOR with more crystal penetration are sometimes adopted to illustrate different PSF kernel sizes between central and off-central LORs. PSF can achieve both (transaxial) uniformity and improvement in spatial resolution.

In this regard, PSF can address the limited spatial resolution and also resolve the partial volume effects (PVEs) that affect both the visual and quantitative analysis of PET [39]. Therefore, PSF-based iterative reconstruction is considered to have the most significant impact on SUV measurements. For example, PSF-based reconstruction without postsmoothing can increase SUVmax by approximately $70 \%$ in small lesions of breast cancer [40] and lung cancer [41].

However, there is another factor of image resolution degradation: the DOI effect, which is the increase in the LOR location uncertainly mostly due to inter-crystal penetration. Because preclinical imaging has a significantly small relative bore size (compared with the body size of small animals) for a larger solid angle than human PET, this problem becomes more significant as the bore size decreases and the solid angle increases, and there may be a higher probability of an off-centered LOR from distant crystals. With whole-body PET as the method of choice in the near future, DOI correction is a challenge in both preclinical and clinical PET imaging.

\section{2) PVC}

Similar to PSF modeling, PVC can be implemented in both reconstruction and post-reconstruction steps. In addition, there is post-reconstruction-based PSF modeling, such as Philips PSF reconstruction (whereas reconstructionbased PSF modeling is for Siemens HD PET and GE SharpIR).

As discussed in a systematic review by Erlandsson et al. [42], there are a variety of PVC methods. In a comprehensive review by Rousset et al. [43], many useful PVC methods were summarized for the combination of post-reconstruction-based/reconstruction-based methods and regionbased/voxel-based methods. These methods include the classical recovery coefficient method [44], region-based method of the geometric transfer matrix (GTM) $[45,46]$, voxel-based methods of iterative deconvolution with noise constraints [47-50], and a Bayesian approach [51]. Because the Bayesian approach can penalize noise by a priori knowledge in an iterative reconstruction pipeline, it will be addressed in the section '2) MAP algorithm'. Recently, a voxel-wise version of the GTM method called the regionbased voxelwise (RBV) PVC method and its extension have been frequently used in literature [23,52] and Freesurfer 6.0.

In a systematic review of PVC in oncology by Cysouw et al. [53], partial volume (PV)-corrected PET may be used as an adjunct (i.e., neither a replacement for PV-uncorrected PET nor a routine application of PVC in standard clinical practice). One major reason for the adjunctive use may be the variety of PVC methods and that oncology PET is usually PV-corrected with voxel-based iterative methods or approximated recovery coefficients estimated by a simpler structure (e.g., sphere).

In contrast, GTM- and RBV-based approaches have been widely used in neuroimaging (in particular, for ADNI cohort PET image analysis) in conjunction with proper cortical parcellation (e.g., Freesurfer software). However, more attention and effort should be paid to potential anatomical image segmentation/registration errors and tissue inhomogeneity [43]. As mentioned in a systematic review by 
Jungsu S. Oh : Nuclear Medicine Physics: Review of Advanced Technology

Erlandsson et al. [42], additional effort should also be made for non-uniform regions $[52,54]$ and correction for tissue fraction $[55,56]$.

For more detailed information, the reader is referred to systematic reviews by Erlandsson et al. [42], Rousset et al. [43], and Cysouw et al. [53].

\section{Iterative reconstruction algorithms}

\section{1) Expectation-maximization algorithm}

There are conventional iterative reconstruction algorithms of the maximum-likelihood expectation-maximization (MLEM) algorithm and its modification for faster convergence called ordered-subset expectation-maximization (OSEM). This class of methods exhibits significant image quality improvement compared with classical analytical methods, such as filtered back-projection.

Expectation-maximization (EM) methods can improve the recovery coefficient of the SUV of lesions as the number of iterations (or iterations $\times$ subsets in OSEM) is increased. However, there is a well-known trade-off between the spatial resolution and SNR. Specifically, the image noise level increases with increased iterations, decreasing small lesion detectability and increasing false positive findings.

Due to the flexible structure of iterative reconstruction pipelines, these methods can be optimized in terms of the trade-off of quantitative accuracy and visual lesion detectability for best clinical practice. Specifically, we can reduce the image noise by compromising the SUV accuracy (e.g., slight SUV underestimation in small lesions) by early-stopping iterations.

\section{2) MAP algorithm}

As mentioned above, the PSF and increased number of iterations can significantly increase SUV-related quantitative metrics in PET. Due to the goal of PSF iterative reconstruction, reconstructed PET images are usually used as is (with no filtering or all-pass filtering) or with relatively small kernel-based smoothing. To resolve both the potential degradation of SNR and incomplete recovery by early termination by PSF iterative reconstruction, Bayesian penalized likelihood (BPL) iterative reconstruction (a class of MAP algorithms), and the PVC method [51] have recently been suggested to improve tumor detection and recovery coefficients while maintaining the stability of SUV metrics.

As the name $M A P / B P L$ implies, this class of methods takes advantage of prior knowledge of the image for the noise penalty function (e.g., non-negativity, and small image intensity changes between adjacent voxels), such as BPL iterative reconstruction marketed as Q.Clear by GE [39]. As a result, this class of methods permits effective convergence of image accuracy by suppressing noise [38,51]. In this regard, it is not necessary to comprise the iterative reconstruction by early termination due to its robustness against noise, that is, high recovery coefficient with low noise. However, physicians sometimes feel that the resulting images include artificial patterns. Therefore, many institutions that employ MAP reconstruction also perform tandem OSEM reconstruction.

\section{NMP technique for SUV harmonization}

\section{1) SUV harmonization problems}

Although the development of NMP has led to better image quality in a TOF-based and PSF-capable PET scanner, there is an unwanted side effect of not being able to directly compare the quantitative measures between PET camera generations (i.e., non-TOF/non-PSF generation vs. TOF/ PSF generation).

Indeed, there are a number of physical factors for the SUV measurement error and difference [57-60] during the entire imaging process. Specifically, these factors include image acquisition/reconstruction parameters, region-of-interest (ROI) or VOI methods, SUV normalization factors (body weight, lean body mass, or liver SUV-based SUV ratio), and image resolutions dominated by PET camera differences (in particular, depending on whether PSF is applied).

\section{2) SUV harmonization solutions}

Among many NM societies, the European Association of Nuclear Medicine (EANM) and EANM Research Ltd. (EARL) established a systematic harmonization approach, as reviewed by Boellaard et al. [57-59] and Aide et al. [60]. In this review, among the technical causes of errors and differences in SUV calculation, reconstruction variability has been noted as a prominent factor in the past decades due 
to the aforementioned software/hardware improvements in NMP.

Above all, the significantly increased spatial resolution by present PSF corrections is considered the most important factor affecting the scanner differences, making direct comparison more difficult. To better handle the camera differences, EANM suggested producing two reconstructions: one optimized for maximum lesion detection and the other optimized for quantitative analysis [61]. However, performing two reconstructions for every PET/CT scan may be not clinically feasible.

Therefore, there have been several approaches for postreconstruction-based SUV harmonization. Most harmonization is based on identifying the optimal smoothing kernel for post-reconstruction that matches the recovery coefficient (and bias) of higher-resolution scanners to that of lower resolution scanners.

Joshi et al. [62] performed brain PET image harmonization on the United States Alzheimer Disease Neuroimaging Initiative (US-ADNI) by comparing the optimal smoothing kernel for matching the digital (i.e., bitmap-based) 3D Hoffman phantom to the corresponding PET image. Thereby, one can achieve brain PET SUV harmonization between various PET camera generations. Conversely, this information can be also used for PVC by considering the spatial blurring of real (PVE-free) uptake as the measured PET uptake $[23,52]$.

For whole-body PET, there are a number of studies using anthropomorphic phantoms with a hot cylinder or spheres for post-reconstruction-based recovery-coefficientmatching harmonization [61,63-66]. To this end, the NEMA IQ phantom or Jaszczak cylindrical phantom (equipped with small hot spheres/cylinders) is frequently used with various lesion-to-background ratios (e.g., 2.5:1-8:1). A dedicated post-reconstruction algorithm (marketed as EQfilter; Siemens Healthineers, Erlangen, Germany) and other third-party tools are also available. There is a report of $<7 \%$ between-scanner differences by both harmonization methods of post-reconstruction-based methods and doublereconstruction methods [61].

\section{NMP Advances in Detector Elements}

\section{Development of detector elements in PET (LSO and LYSO)}

LSO was first used in small-animal PET [67], in a brain scanner [68,69], and in a whole-body PET scanner [70,71]. As mentioned above, LSO was in the center of TOF PET development due to its superior characteristics in terms of sensitivity, spatial resolution, reduced deadtime (or fast decay time), and random coincidences. Fast photomultiplier tubes (PMTs) and improved electronics led to practical TOF PET systems [72,73]. As mentioned above, LSO- and LYSObased TOP PET finally became the standard PET method in the 2000s [16-19].

\section{Development of detector elements in digital PET and PET/MR (SiPM)}

The SiPM will be addressed in PET/MR and Digital PET.

\section{Development of detector elements in gamma camera/SPECT (cadmium zinc telluride)}

Whereas PET imaging has made a breakthrough with TOF/PSF and SiPM-based digital PET and PET/MR, most gamma camera detectors remained with an $\mathrm{NaI}(\mathrm{Tl})$-based conventional system for many decades. Recently, the cadmium zinc telluride (CZT) solid-state detector-based SPECT camera has emerged and shown potential strength in physical performance, including high sensitivity and spatial resolution, outperforming conventional gamma camera scintillators in terms of energy resolution (i.e., better scatter rejection performance), and high count rate capability ( $>100$ million photons $/ \mathrm{s} / \mathrm{mm}^{2}$ ). CZT is a room-temperature semiconductor detector material that directly converts gamma (and X-ray) photons into electrons and holes.

Moreover, the quantification of myocardial perfusion is an emerging topic in nuclear cardiology with an expected diagnostic/prognostic incremental value, especially in coronary artery disease. Dynamic myocardial perfusion imaging-based coronary flow reserve or myocardial flow 
Jungsu S. Oh : Nuclear Medicine Physics: Review of Advanced Technology

reserve are known to be associated with adverse cardiovascular events independent of luminal angiographic severity [74]. The advent of a new dedicated CZT-based camera created a new opportunity for perfusion quantification in myocardial SPECT. In addition, small-organ imaging, such as breast and brain imaging, has also been attempted.

\section{NMP in Hybrid/Advanced Imaging}

\section{PET/CT}

Since the initial integration of PET/CT by Townsend (2008), hybrid PET/CT imaging has become crucial for clinically feasible routine PET imaging free from the timeconsuming transmission PET scan, allowing PET to afford more efforts for emission scan. Moreover, PET/CT allows for essential multimodality imaging, providing both anatomical and metabolic information for oncology, neurology, and cardiology $[15,22]$. Now that PET/CT has become the standard and most common modality of PET imaging and has been installed worldwide, PET usually refers to PET/CT.

\section{PET/MR and digital PET using SiPM}

Thanks to the soft-tissue contrast of MR and tremendous technical advances in both PET and MR, the development of hybrid PET/MR systems has been a major focus in the past decade. However, there have been several challenges in the development of PET/MR [75,76]. First, conventional PET photodetectors (i.e., devices for multiplication of light produced by scintillation crystals) are based on PMTs that are susceptible to the strong magnetic fields used in the MR scanner. To avoid this problem, the first integrated PET/ MR was implemented using a type of solid-state semiconductor-based photodetector called avalanche photodiodes (APDs). However, APDs (and SiPMs) have much smaller physical dimensions than PMTs; therefore, the remaining bore size $(\sim 60 \mathrm{~cm})$ after PET was inserted in the MR was sufficient for patient scanning.

Whereas ordinary APDs have limited amplifier gain and poor timing resolution ( $2 \mathrm{~ms})$ and are thus unsuitable for TOF PET, Geiger-mode APDs (also known as SiPMs) are relatively free from these limitations. Therefore, SiPMs have become the current standard for PET/MR photodetectors.

Attenuation correction (AC) is another major physical problem in PET/MR, in contrast to CT-based AC, which is straightforward in older PET/CT systems. AC of bone has remained challenging for many years not only because bone is the critical structure of attenuation of the PET emission signal, but also because it has very low proton density (and thus a very low MR signal).

Several approaches have been proposed, including dedicated fast MR sequence (e.g., Dixon sequence), ultra-short time-to-echo (UTE)/zero time-to-echo (ZTE) MR, and a combination of both, and TOF-based AC [77-80]. However, Dixon sequence-based AC may suffer from bone AC error ( $>10 \%$ AC error and $>30 \%$ quantification error in osseous structures adjacent to bone $[75,81])$ due to assigning the same AC coefficients for bone and soft tissue. In addition, UTE/ZTE MR suffers from severe noise.

Alternatively, we can use atlas-based methods [76,82] of retrospectively assigning bone information to the MR AC map. However, this approach has the potential limitation in that it requires previous knowledge for generating the bone template and an appropriate spatial registration (more specifically, spatial normalization and inverse normalization) tool.

In addition, there are potential lung segmentation errors, unrealistic uniform AC, and MR FOV truncation. To fully understand the limitations and perspectives of current PET/MR technology, readers are referred to a systematic review by Boellaard and Quick [75]. The author believes that these challenges can be overcome by DL approaches, as will be discussed in the final section.

Finally, digital SiPM (near one-to-one coupling of SiPM and crystal) has recently been introduced for commercialized digital PET (e.g., GE Signa PET/MR and Discovery MI PET/CT with SiPM, Siemens mMR with APD and Vision PET/CT with SiPM, Philips Vereos with SiPM). Thanks to near one-to-one coupling (2:1-1:1), digital PET can achieve improved spatial resolution and time resolution, permit high maximum count rates [83-85], and demonstrate higher image quality that can also lead to dose reduction $[86,87]$. 


\section{Whole-body PET}

Whole-body PET has already been described in NMP Advances in Image Acquisition/Reconstruction section.

\section{NMP in Precision Medicine}

\section{NMP in theranostics (also known as theranosis)}

\section{1) Theranostics and personalized medicine}

Molecular imaging-based personalized/precision medicine and a key technology called theranostics have been a well-established and important concept for individualized therapeutic strategies [88]. To avoid unnecessary/expensive treatments and to optimize patient treatment in terms of target localization and treatment methods, personalized medicine has been a major focus in recent decades [88].

Although theranostics molecular imaging (i.e., RAI-131) was introduced long ago (first performed in 1946), the term "theranostics", which has been used in recent decades (first used in the early 1990s), refers to a technique for a specific molecular-targeted therapy by combined diagnostic testing and therapeutic modalities, mostly based on PET or SPECT diagnostic imaging using the same molecule with therapeutics or a very similar molecule (also known as an analogue) as a therapeutic radiopharmaceutical [88-90].

Theranostics is performed mainly for individualized therapeutic decision-making, for attaining high efficacy, and for reducing adverse effects in a patient-specific manner. In this regard, theranostics is the epitome and key technology of personalized medicine [88].

Diagnostic imaging-based visualization/localization of therapeutic targets indicating which sites require treatment (diagnostic scan [90]) can help treatment outcome prediction and monitoring, confirming that the sites have been treated (post-therapy scan [90]), as well as help determine the best treatment strategy. By its nature, a tumor is heterogeneous within a subject as well as between subjects, and there are thus many challenges in the execution of targeted therapy in cancer [90]. In this context, a whole-body imaging-based approach may be beneficial; for example, a single tumor biopsy cannot capture all tumor heterogeneity without underestimating the genetic mutational burden, subsequently contributing to treatment failure or drug resistance [88-90].

As the medical specialty of theranostics is well established as a molecular imaging-based personalized (i.e., specific molecular-targeted) therapy and has been for three-quarters of a century [88], we must focus on this new area of NMP. Theranostics for thyroid by RAI has been performed for differentiated thyroid cancer, contributing to modern tailored personalized management by providing a high therapeutic effect and by avoiding significant adverse effects $[88,91,92]$. SPECT/CT, which can provide the SUV by appropriate sensitivity calibration, can significantly improve the precision and sensitivity of whole-body iodine scintigraphy and in turn contribute to improved precision medicine [90].

Recently, somatostatin receptor-based theranostics has been studied for neuroendocrine tumors (NETs), for example, Lu-177 DOTATOC and DOTATATE-based treatment in conjunction with Ga-68-based diagnostic imaging [89]. In South Korea, several institutions have also established or practiced theranostics of NETs. In addition, Lu-177-based SPECT/CT imaging can be used for radiation dosimetry for assessing the therapeutic efficiency and unwanted radiation exposure to critical organs (e.g., kidney/bladder/red marrow).

For metastatic prostate cancer, for example, we have prostate-specific membrane antigen (PSMA) diagnostic ligands, including Ga-68 PSMA series for PET imaging and therapeutic ligands, including Lu-177 J-591 and Lu-177-PSMA-617 [89].

\section{2) NMP perspective: dosimetry for theranostics}

Because theranostics other than RAI is at the beginning stage, its role in NMP is not yet clear. However, the aforementioned internal dosimetry (e.g., Lu-177-based SPECT/ CT for Lu-177 DOTATOC or DOTATATE-based treatment) has become much more important than conventional diagnostic PET or SPECT imaging due to its direct association with therapeutics per the nature of theranostics. General dosimetry may require whole-body organ delineation and multiple serial whole-body PET scans [93]. However, this may be intractable as routine practice for theranostics due to the much longer half-life of the therapeutic radioisotope 
(e.g. Lu-177) than of F-18. Recently, the single time imagingbased dosimetry method [94] has reported low variability from more rigorous methods (i.e., multi-time-point-based exponential fitting for residence time calculation).

Because internal dosimetry is closely related to the estimation of external radiation, dosimetry may be the main NMP part in theranostics. Thanks to the development of related DL technique, we can also consider automatic delineation of target lesions and critical organs.

\section{2. $\mathrm{DL}$ in NM precision medicine}

1) Artificial intelligence, machine learning, and $D L$

Before discussing DL in NM, Artificial intelligence (AI)/ machine learning (ML)/DL must be explained. DL is a specific type of ML using a deep network (i.e., artificial neural network [ANN] with more than one hidden layer), and both are AI concepts [95]. For the history of ML, common algorithms, and reasons for using machine learning, readers are referred to the systematic review by Uribe et al. [96], which emphasizes traditional ML techniques, including naïve Bayes classification, linear regression, support vector machine (SVM), random forest, principal component analysis, k-means/hierarchical clustering, and ANN.

Although the principles of AI and ML have been proposed much earlier (first described in 1956), their practical implementation was delayed by several decades. In particular, DL suffered from a larger delay because higher computing power and sophisticated training algorithms [97] were required for the significantly larger number of training parameters than for the traditional ML algorithm.

In traditional medical image processing (as other biomedical research), only a specific algorithm provided by humans was used for learning features from medical imaging. Therefore, the performance was highly dependent on the domain knowledge and expertise of the user or programmer. In $\mathrm{AI} / \mathrm{ML}$, computers can learn features from observations (e.g., medical imaging data) based on a mathematical model and cost function by training unknown variables (e.g., weights in ANN and SVM), and further make statistical inferences from learning [96].

Unlike traditional ML, DL can learn features from data by training a deep ANN (in NM imaging, mostly based on a convolutional neural network [CNN]) without or with very little domain knowledge. Each layer of the CNN has multiple channels of convolution kernels that can learn specific filters that can extract hidden features from an image. As the layer becomes deeper, the extracted information is integrated into a higher level of the hierarchy (or larger scale) information. Thereby, deep learning can extract a large amount of useful information for classification, regression by convolution-based encoding, and image generation by further transposed convolution-based decoding (e.g., UNET).

For more information on $\mathrm{AI} / \mathrm{ML}$, readers are referred to the aforementioned reviews by Visvikis et al. [95] and Uribe et al. [96]. For more information on DL, readers are referred to reviews by Visvikis et al. [95] and Choi [98].

\section{2) Rise of $M L / D L$ algorithms}

The amount of biomedical data (medical imaging and radiomics/radiogenomic-related data) is rapidly increasing, thus forming availability of big data, which is the key ingredient for training ML and is particularly important for DL $[98,99]$. Advances in the theory of stochastic gradient (in an analogy of MLEM and OSEM) [97] made it possible to train ML with big data by faster convergence, which enabled us to train DL with a more complex structure more efficiently. Computing hardware development (including the central processing unit [CPU], graphics processing unit [GPU], and neural network Tensor Processing Unit [TPU] [35]) made it possible to train ML/DL epochs with big data, especially for sufficient epochs to converge the DL network.

\section{3) $M L / D L$ algorithms in medical imaging}

Many systematic reviews [95-98] have indicated that recent advances in DL have impacted various scientific fields and demonstrated successful results in many areas. In particular, there is growing research and clinical interest in automatic medical image analysis, including radiomics and radiogenomics [100-116] that may reveal additional information that is not accessible even by experts [95].

\section{4) $\mathrm{ML} / \mathrm{DL}$ algorithms in NM imaging}

As radiomics and radiogenomics have been the most representative approach for imaging-based precision medicine 
in recent decades, the main objective of this evolution (also known as Industry 4.0) may be to assign medical imaging a larger role [95] as follows. Medical imaging should produce more useful information from imaging studies for precision medicine. More importantly, NM imaging should move beyond diagnosis (as addressed in the section of Theranostics) to be an essential integrative part covering all clinical decision-making systems (e.g., therapy planning/monitoring/assessment, predictive modeling, and stratification). With sufficient understanding of the rationale of DL in NM, the author would like to discuss more specific applications as follows.

\section{5) DL application in NM 1: classification}

Classification is one of the oldest and most extensively researched ML areas in NM (e.g., disease group classification or diagnosis/differential diagnosis, including automatic diagnosis of neurodegenerative diseases, such as Alzheimer's disease [24,107,108] and Parkinson's disease [109], and brain perfusion reserve decreases [110]). These methods can be easily extended for precision medicine, as the promising diagnostic accuracy (area-under-the-curve $>0.9$ ) may lead to automatic classification of the genotype and phenotype of tumors with higher accuracy than conventional methods, thus aiding decision-making for personalized medicine in an extended line of recent radiomics and radiogenomics studies [100-106].

6) DL application in NM 2: automatic segmentation and quantification

Precise and accurate automatic segmentation of lesion VOIs is a prerequisite not only for quantitative analysis, but also for precision medicine, as the accuracy of VOI can dominate the accuracy of subsequent quantification and decision-making. Indeed, many useful radiomics and radiogenomics features are extracted from well-delineated VOIs [100-106].

Although manual delineation may be the gold standard for this task, it is labor-intensive to delineate many VOIs for research or routine quantification purposes. Moreover, differences between raters and their expertise may lead to high inter-rater variability. CNN-based DL is considered one of the best solutions for accuracy and inter-rater reli- ability. Therefore, there have been a number of CNN-based segmentation studies for PET or CT segmentation for quantitative PET analysis [111-113], and CT segmentation for quantitative SPECT analysis [114]. Interestingly, U-NET has been extensively used in most studies as the generator for automatic segmentation. This may be due to the strength of U-NET in the automatic encoding of multi-scale features by combinations of convolution and strided-pooling layers, and in residual learning by skip connection between the encoding and decoding parts of the CNN.

\section{7) DL application in NM 3: image generation}

Image generation is a unique area of DL in which traditional ML technology and/or radiomics/radiogenomics technology cannot compete. Although the ordinary structure of a CNN may be sufficient for generating a binary mask of VOI segmented, recent image generation tasks are more complex (e.g., increasing SNR of low-dose PET $[115,116]$, increasing spatial resolution of PET [117], and generating an MR-like mask from amyloid PET [118]). In this situation, a generative adversarial network (GAN) [104] architecture is useful and has thus been used by many previous studies [115-118]. It should be noted that the conditional GAN, which uses a realistic image as the input for CNN unlike the original (noise-based) GAN [97], is used for the purpose of medical image generation. Thereby, image generation can solve many of the aforementioned complex and seemingly impossible problems by conventional signal processing and ML on the basis of big data.

Interestingly, DL-based image generation can also be used to help image reconstruction. There have been several studies using U-NET or autoencoder-based CNN for generating a CT AC map using a TOF-based MLAA map not only for brain PET [119], but also for whole-body PET [120]. In addition, Gong et al. [121] performed Dixon and UTE MRbased AC map generation for PET/MR.

\section{Discussion and Perspectives}

\section{Limitations of this review for in vitro NM}

In addition to NM imaging, in vitro NM (mostly based on radioligand binding assay or radioimmunoassay) is worthy 
Jungsu S. Oh : Nuclear Medicine Physics: Review of Advanced Technology

of consideration as another component of NM. Although there also are NMP-related parts, including the gamma counting-based system in in vitro NM, they are not addressed in this review for the sake of brevity.

\section{Limitations of this review on tracer kinetic modeling}

Tracer kinetic modeling (TKIN) is a promising technique for NMP and NM image processing that can provide quantitative kinetic model parameters and that has an enormous number of subfields, including graphical analyses and tissue compartment models. In this context, the SUV is sometime called a semi-quantitative measure, even though it is quantitative measure of molecular imaging that other modalities cannot achieve.

Although they are quantitative by nature, NM images in most clinical publications and routine practice have been analyzed mostly visually or by the SUV [95], largely due to difficulties of performing TKIN in routine clinical practice. In this context, this important area of NMP is not discussed in the main text.

However, routine TKIN may become a key part of NM/ NMP in the near future thanks to whole-body PET and PET/ MR, as noted by Cherry et al. [36] and Cabello and Ziegler [76]. Moreover, DL-based and/or PET/MR-based automatic delineation of VOI also has potential to facilitate subsequent TKIN (e.g., one for arterial input function extraction [76]).

\section{Convergence between multiple disciplines and multiple centers}

As noted by Jones [13], to meet the goals of converging molecular imaging with molecular biology and molecular medicine, we must collaborate between multiple disciplines and between academia and industries. Moreover, randomized/controlled multicenter clinical trials are now requisite for the successful development of pharmaceutical and therapeutic methods for patient healthcare.

As mentioned by Aide et al. [60], NM and NMP societies should focus on ensuring exchangeability or pooling of quantitative results in a multicenter setting for this purpose, which can also be beneficial to derive diagnostic criteria for routine PET/CT metrics. In this context, NM imaging standardization and harmonization is a crucial step for the Korean NMP society as well and will be discussed in the next section.

Theranostics and DL may be pivotal in current NM and NMP. In this context, multidiscipline convergence and multicenter clinical trials will facilitate theranostics and DL, both of which demand (well-labeled) big data. Now that many Korean studies have also been published in this area, the Korean NMP society should exert more effort in contributing to Korean NM/MP, precision medicine, and patient welfare.

\section{Standardization, harmonization, and beyond}

As mentioned, EANM and EARL have established systematic reviews/guidelines/ systems for multicenter NM image standardization and harmonization. These developments have influenced oncological/neurological/cardiological diagnosis and disease staging, as well as therapy response monitoring and planning [60].

In addition, greater efforts by the NMP society for quality control/quality assurance (QC/QA) of NM imaging should be made. Indeed, most of the aforementioned standardization and harmonization of NM imaging is inevitably related to QC/QA, including calibration and corrective action for QC errors. Moreover, many useful metrics for NM imaging harmonization are from routine QC/QA (e.g., NEMA/ACR phantom-based quarterly QC for recovery coefficient measurements).

In this context, Korean NMP society members should also benchmark themselves with the American College of Radiology (ACR) accreditation and EANM guidelines and EARL accreditation for FDG PET/CT tumor imaging, and should align with them and proceed to establish their own accreditation system.

Based on the aforementioned guidelines, EANM/EARL launched a PET/CT QC program in 2010 aiming to harmonize image quality and quantification across sites and cameras [60]. Both the EARL program and ACR accreditation recommend using a specific set of QC experiments. EARL accreditation established the acceptance limits for SUV bias 
and recovery coefficients based on the results of a feasibility study performed on PET/CT systems currently used in clinical practice. Similar PET scanner qualification is also performed as part of the ACR accreditation and in ACRIN PET scanner qualification.

At present, many Korean institutions are accredited or qualified by these systems; for example, one Korean institution (Asan Medical Center) is continually accredited by both ACR PET accreditation and ACR NM accreditation, and several institutions are continually qualified for their PET scanner by ACRIN PET qualification. In addition, many Korean studies on standardized/harmonized NM imaging data have also been published in this important area. Therefore, it is time for the Korean NMP society to establish the perspective for the PET/NM center accreditation and PET/SPECT scanner qualification.

\section{Concluding Remarks}

In this paper, the author provided a brief and comprehensive review of advanced technologies of NMP. The advances are in various areas of NMP, including image acquisition/ reconstruction, detector material/devices, hybrid/advanced imaging, precision medicine, and DL, and have led to highquality digital imaging of invaluable in vivo whole-body molecular information for NM and NMP. Owing to these strengths and currents trends of convergence/consilience with other imaging modalities and disciplines, the author believes that NMP can contribute to enhanced image quality and decreased radiation exposure, and consequently, quantitative and personalized healthcare.

\section{Acknowledgements}

It is a great honor for the author to have this opportunity to write a nuclear medicine physics review article for the 30th Anniversary Series in PMP. The publication of this article was supported by PMP and the Korean Society of Medical Physics (KSMP).

\section{Conflicts of Interest}

The authors have nothing to disclose.

\section{Availability of Data and Materials}

All relevant data are within the paper and its Supporting Information files.

\section{References}

1. Cherry SR, Phelps ME, Sorenson JA. Physics in nuclear medicine. 4th ed. Philadelphia: Saunders/Elsevier; 2012.

2. Anderson CJ, Ling X, Schlyer DJ, Cutler CS. A short history of nuclear medicine. Radiopharmaceutical chemistry. Cham: Springer; 2019:11-26.

3. Wagner HN Jr. A brief history of positron emission tomography (PET). Semin Nucl Med. 1998;28:213-220.

4. L'Annunziata MF. Radioactivity: introduction and history. Amsterdam: Elsevier; 2007.

5. Hertz S, Roberts A. Radioactive iodine in the study of thyroid physiology; the use of radioactive iodine therapy in hyperthyroidism. J Am Med Assoc. 1946;131:81-86.

6. McCready VR. Milestones in nuclear medicine. Eur J Nucl Med. 2000;27(1 Suppl):S49-S79.

7. Ansell G, Rotblat J. Radioactive iodine as a diagnostic aid for intrathoracic goitre. Br J Radiol. 1948;21:552-558.

8. Cassen B, Curtis L, Reed CW. A sensitive directional gamma-ray detector. Oak Ridge, USA: United States Atomic Energy Commission. 1950; UCLA-49. 78-81 p.

9. Mallard J, Trott NG. Some aspects of the history of nuclear medicine in the United Kingdom. Semin Nucl Med. 1979; 9:203-217.

10. Mallard JR. Hevesy memorial medal lecture 1985. Some call it laziness: I call it deep thought (with apologies to Garfield). Nucl Med Commun. 1987;8:691-710.

11. Kuhl DE, Edwards RQ. Reorganizing data from transverse section scans of the brain using digital processing. Radiology. 1968;91:975-983.

12. Nellemann P, Hines H, Braymer W, Muehllehner G, Geagan M. Performance characteristics of a dual head SPECT scanner with PET capability. IEEE Trans Nucl Sci. 1995;3: 1751-1755.

13. Jones T. The imaging science of positron emission tomography. Eur J Nucl Med. 1996;23:807-813.

14. Moses WW. Fundamental limits of spatial resolution in PET. Nucl Instrum Methods Phys Res A. 2011;648 Supple- 
ment 1:S236-S240.

15. Townsend DW. Combined positron emission tomography-computed tomography: the historical perspective. Semin Ultrasound CT MR. 2008;29:232-235.

16. Surti S, Kuhn A, Werner ME, Perkins AE, Kolthammer J, Karp JS. Performance of Philips Gemini TF PET/CT scanner with special consideration for its time-of-flight imaging capabilities. J Nucl Med. 2007;48:471-480.

17. Karp JS, Surti S, Daube-Witherspoon ME, Muehllehner G. Benefit of time-of-flight in PET: experimental and clinical results. J Nucl Med. 2008;49:462-470.

18. Jakoby BW, Bercier Y, Conti M, Casey ME, Bendriem B, Townsend DW. Physical and clinical performance of the mCT time-of-flight PET/CT scanner. Phys Med Biol. 2011; 56:2375-2389.

19. Bettinardi V, Presotto L, Rapisarda E, Picchio M, Gianolli L, Gilardi MC. Physical performance of the new hybrid PET/CT Discovery-690. Med Phys. 2011;38:5394-5411.

20. Lecoq P. Pushing the limits in time-of-flight PET imaging. IEEE Trans Radiat Plasma Med Sci. 2017;1:473-485.

21. Berg E, Cherry SR. Innovations in instrumentation for positron emission tomography. Semin Nucl Med. 2018;48: 311-331.

22. van der Vos CS, Koopman D, Rijnsdorp S, Arends AJ, Boellaard R, van Dalen JA, et al. Quantification, improvement, and harmonization of small lesion detection with state-ofthe-art PET. Eur J Nucl Med Mol Imaging. 2017;44(Suppl 1): 4-16.

23. Son HJ, Oh JS, Roh JH, Seo SW, Oh M, Lee SJ, et al. Differences in gray and white matter ${ }^{18} \mathrm{~F}$-THK5351 uptake between behavioral-variant frontotemporal dementia and other dementias. Eur J Nucl Med Mol Imaging. 2019;46: 357-366.

24. Son HJ, Oh JS, Oh M, Kim SJ, Lee JH, Roh JH, et al. The clinical feasibility of deep learning-based classification of amyloid PET images in visually equivocal cases. Eur J Nucl Med Mol Imaging. 2020;47:332-341.

25. Wahl RL, Jacene H, Kasamon Y, Lodge MA. From RECIST to PERCIST: evolving considerations for PET response criteria in solid tumors. J Nucl Med. 2009;50 Suppl 1(Suppl 1): 122S-150S.

26. Strother SC, Casey ME, Hoffman EJ. Measuring PET scanner sensitivity: relating countrates to image signal-to- noise ratios using noise equivalents counts. IEEE Trans Nucl Sci. 1990;37:783-788.

27. Campagnolo RE, Garderet P, Vacher J. Tomographie par emetteurs positrons avec mesure de temps de vol. Colloque national sur le traitement du signal. Nice: Rennes Cedex; 1979.

28. Vandenberghe S, Mikhaylova E, D'Hoe E, Mollet P, Karp JS. Recent developments in time-of-flight PET. EJNMMI Phys. 2016;3:3.

29. Ter-Pogossian MM, Mullani NA, Ficke DC, Markham J, Snyder DL. Photon time-of-flight-assisted positron emission tomography. J Comput Assist Tomogr. 1981;5:227-239.

30. Laval M, Gariod R, Allemand R, Cormorèche E, Moszynski M. The "LETI" positron tomograph architecture and time-of-flight improvements. Paper presented at: Workshop on Time of Flight Tomography; 1982 May 17-19; St Louis, USA.

31. Yamamoto M, Ficke DC, Ter-Pogossian MM. Experimental assessment of the gain achieved by the utilization of timeof-flight information in a positron emission tomograph (Super PETT I). IEEE Trans Med Imaging. 1982;1:187-192.

32. Budinger TF. Time-of-flight positron emission tomography: status relative to conventional PET. J Nucl Med. 1983; 24:73-78.

33. Wong WH. PET camera performance design evaluation for BGO and BaF2 scintillators (non-time-of-flight). J Nucl Med. 1988;29:338-347.

34. Mallon A, Grangeat P. Three-dimensional PET reconstruction with time-of-flight measurement. Phys Med Biol. 1992;37:717-729.

35. Patterson D. 50 years of computer architecture: from the mainframe CPU to the domain-specific tpu and the open RISC-V instruction set. Paper presented at: 2018 IEEE International Solid - State Circuits Conference - (ISSCC); 2018 Feb 11-15; San Francisco, USA.

36. Cherry SR, Jones T, Karp JS, Qi J, Moses WW, Badawi RD. Total-body PET: maximizing sensitivity to create new opportunities for clinical research and patient care. J Nucl Med. 2018;59:3-12.

37. Badawi RD, Shi H, Hu P, Chen S, Xu T, Price PM, et al. First human imaging studies with the EXPLORER total-body PET scanner. J Nucl Med. 2019;60:299-303.

38. Panin VY, Kehren F, Michel C, Casey M. Fully 3-D PET 
reconstruction with system matrix derived from point source measurements. IEEE Trans Med Imaging. 2006;25: 907-921.

39. Takamochi K, Yoshida J, Murakami K, Niho S, Ishii G, Nishimura M, et al. Pitfalls in lymph node staging with positron emission tomography in non-small cell lung cancer patients. Lung Cancer. 2005;47:235-242.

40. Bellevre D, Blanc Fournier C, Switsers O, Dugué AE, Levy C, Allouache D, et al. Staging the axilla in breast cancer patients with ${ }^{18}$ F-FDG PET: how small are the metastases that we can detect with new generation clinical PET systems? Eur J Nucl Med Mol Imaging. 2014;41:1103-1112.

41. Kuhnert G, Boellaard R, Sterzer S, Kahraman D, Scheffler M, Wolf J, et al. Impact of PET/CT image reconstruction methods and liver uptake normalization strategies on quantitative image analysis. Eur J Nucl Med Mol Imaging. 2016;43:249-258.

42. Erlandsson K, Buvat I, Pretorius PH, Thomas BA, Hutton BF. A review of partial volume correction techniques for emission tomography and their applications in neurology, cardiology and oncology. Phys Med Biol. 2012;57:R119R159.

43. Rousset O, Rahmim A, Alavi A, Zaidi H. Partial volume correction strategies in PET. PET Clin. 2007;2:235-249.

44. Hoffman EJ, Huang SC, Phelps ME. Quantitation in positron emission computed tomography: 1. Effect of object size. J Comput Assist Tomogr. 1979;3:299-308.

45. Rousset O, Ma Y, Kamber M, Evans AC. 3D simulations of radiotracer uptake in deep nuclei of human brain. Comput Med Imaging Graph. 1993;17:373-379.

46. Rousset OG, Ma Y, Evans AC. Correction for partial volume effects in PET: principle and validation. J Nucl Med. 1998;39:904-911.

47. Schafer RW, Mersereau RM, Richards MA. Constrained iterative restoration algorithms. Proc IEEE. 1981;69:432450.

48. Carasso A. Linear and nonlinear image deblurring: a documented study. SIAM J Numer Anal. 1999;36:1659-1689.

49. Rudin L, Osher S, Fatemi E. Nonlinear total variation based noise removal algorithms. Phys D. 1992;60:259-268.

50. Teo BK, Seo Y, Bacharach SL, Carrasquillo JA, Libutti SK, Shukla H, et al. Partial-volume correction in PET: validation of an iterative postreconstruction method with phan- tom and patient data. J Nucl Med. 2007;48:802-810.

51. Green PJ. Bayesian reconstructions from emission tomography data using a modified EM algorithm. IEEE Trans Med Imaging. 1990;9:84-93.

52. Thomas BA, Erlandsson K, Modat M, Thurfjell L, Vandenberghe R, Ourselin S, et al. The importance of appropriate partial volume correction for PET quantification in Alzheimer's disease. Eur J Nucl Med Mol Imaging. 2011;38: 1104-1119.

53. Cysouw MCF, Kramer GM, Schoonmade LJ, Boellaard R, de Vet HCW, Hoekstra OS. Impact of partial-volume correction in oncological PET studies: a systematic review and meta-analysis. Eur J Nucl Med Mol Imaging. 2017;44: 2105-2116.

54. Erlandsson K, Hutton BF. Partial volume correction in SPECT using anatomical information and iterative FBP. Tsinghua Sci Technol. 2010;15:50-55.

55. Boening G, Pretorius PH, King MA. Study of relative quantification of Tc-99m with partial volume effect and spillover correction for SPECT oncology imaging. IEEE Trans Nucl Sci. 2006;53:1205-1212.

56. Lambrou T, Groves AM, Erlandsson K, Screaton N, Endozo R, Win T, et al. The importance of correction for tissue fraction effects in lung PET: preliminary findings. Eur J Nucl Med Mol Imaging. 2011;38:2238-2246.

57. Boellaard R. Standards for PET image acquisition and quantitative data analysis. J Nucl Med. 2009;50 Suppl 1: 11S-20S.

58. Boellaard R. Methodological aspects of multicenter studies with quantitative PET. Methods Mol Biol. 2011;727:335349.

59. Boellaard R. Mutatis mutandis: harmonize the standard! J Nucl Med. 2012;53:1-3.

60. Aide N, Lasnon C, Veit-Haibach P, Sera T, Sattler B, Boellaard R. EANM/EARL harmonization strategies in PET quantification: from daily practice to multicentre oncological studies. Eur J Nucl Med Mol Imaging. 2017;44(Suppl 1):17-31.

61. Ferretti A, Chondrogiannis S, Rampin L, Bellan E, Marzola MC, Grassetto G, et al. How to harmonize SUVs obtained by hybrid PET/CT scanners with and without point spread function correction. Phys Med Biol. 2018;63:235010.

62. Joshi A, Koeppe RA, Fessler JA. Reducing between scan- 
ner differences in multi-center PET studies. Neuroimage. 2009;46:154-159.

63. Oh JS, Kang BC, Roh JL, Kim JS, Cho KJ, Lee SW, et al. Intratumor textural heterogeneity on pretreatment (18) F-FDG pET images predicts response and survival after chemoradiotherapy for hypopharyngeal cancer. Ann Surg Oncol. 2015;22:2746-2754.

64. Kim JW, Oh JS, Roh JL, Kim JS, Choi SH, Nam SY, et al. Prognostic significance of standardized uptake value and metabolic tumour volume on ${ }^{18} \mathrm{~F}$-FDG PET/CT in oropharyngeal squamous cell carcinoma. Eur J Nucl Med Mol Imaging. 2015;42:1353-1361.

65. Ha SC, Oh JS, Roh JL, Moon H, Kim JS, Cho KJ, et al. Pretreatment tumor SUVmax predicts disease-specific and overall survival in patients with head and neck soft tissue sarcoma. Eur J Nucl Med Mol Imaging. 2017;44:33-40.

66. Lim WS, Oh JS, Roh JL, Kim JS, Kim SJ, Choi SH, et al. Prediction of distant metastasis and survival in adenoid cystic carcinoma using quantitative ${ }^{18}$ F-FDG PET/CT measurements. Oral Oncol. 2018;77:98-104.

67. Cherry SR, Shao Y, Silverman RW, Meadors K, Siegel S, Chatziioannou A, et al. MicroPET: a high resolution PET scanner for imaging small animals. IEEE Trans Nucl Sci. 1997;44:1161-1166.

68. Wienhard K, Schmand M, Casey ME, Baker K, Bao J, Eriksson L, et al. The ECAT HRRT: performance and first clinical application of the new high resolution research tomograph. IEEE Trans Nucl Sci. 2002;49:104-110.

69. Schmand M, Eriksson L, Casey ME, Andreaco MS, Melcher C, Wienhard K, et al. Performance results of a new DOI detector block for a high resolution PET-LSO research tomograph HRRT. IEEE Trans Nucl Sci. 1998;45: 3000-3006.

70. Spinks TJ, Bloomfield PM. A comparison of count rate performance for ${ }^{15} \mathrm{O}$-water blood flow studies in the CTI $\mathrm{HR}+$ and accel tomographs in 3D mode. Paper presented at: 2002 IEEE Nuclear Science Symposium Conference Record; 2002 Nov 10-16; Norfolk, USA.

71. Muehllrhner G, Karp JS, Surti S. Design considerations for PET scanners. Q J Nucl Med. 2002;46:16-23.

72. Lewellen TK. Time-of-flight PET. Semin Nucl Med. 1998; 28:268-275.

73. Moses WW, Derenzo SE. Prospects for time-of-flight PET using LSO scintillator. IEEE Trans Nucl Sci. 1999;46:474478.

74. Han S, Kim YH, Ahn JM, Kang SJ, Oh JS, Shin E, et al. Feasibility of dynamic stress ${ }^{201} \mathrm{Tl} /$ rest ${ }^{99 \mathrm{~m}} \mathrm{Tc}$-tetrofosmin single photon emission computed tomography for quantification of myocardial perfusion reserve in patients with stable coronary artery disease. Eur J Nucl Med Mol Imaging. 2018;45:2173-2180.

75. Boellaard R, Quick HH. Current image acquisition options in PET/MR. Semin Nucl Med. 2015;45:192-200.

76. Cabello J, Ziegler SI. Advances in PET/MR instrumentation and image reconstruction. Br J Radiol. 2018;91: 20160363.

77. Hofmann M, Pichler B, Schölkopf B, Beyer T. Towards quantitative PET/MRI: a review of MR-based attenuation correction techniques. Eur J Nucl Med Mol Imaging. 2009; 36 Suppl 1:S93-S104.

78. Mehranian A, Zaidi H. Emission-based estimation of lung attenuation coefficients for attenuation correction in time-of-flight PET/MR. Phys Med Biol. 2015;60:4813-4833.

79. Benoit D, Ladefoged CN, Rezaei A, Keller SH, Andersen FL, Højgaard L, et al. Optimized MLAA for quantitative nonTOF PET/MR of the brain. Phys Med Biol. 2016;61:88548874.

80. Cheng JC, Salomon A, Yaqub M, Boellaard R. Investigation of practical initial attenuation image estimates in TOF-MLAA reconstruction for PET/MR. Med Phys. 2016; 43:4163.

81. Samarin A, Burger C, Wollenweber SD, Crook DW, Burger IA, Schmid DT, et al. PET/MR imaging of bone lesions-implications for PET quantification from imperfect attenuation correction. Eur J Nucl Med Mol Imaging. 2012; 39:1154-1160.

82. Hofmann M, Bezrukov I, Mantlik F, Aschoff P, Steinke F, Beyer T, et al. MRI-based attenuation correction for wholebody PET/MRI: quantitative evaluation of segmentationand atlas-based methods. J Nucl Med. 2011;52:1392-1399.

83. Frach T, Prescher G, Degenhardt C, de Gruyter R, Schmitz A, Ballizany R. The digital silicon photomultiplier- principle of operation and intrinsic detector performance. Paper presented at: 2009 IEEE Nuclear Science Symposium Conference Record (NSS/MIC); 2009 Oct 24-Nov 1; Orlando, USA. 
84. Degenhardt C, Prescher G, Frach T, Thon A, de Gruyter $\mathrm{R}$, Schmitz A, et al. The digital silicon photomultiplier- a novel sensor for the detection of scintillation light. Paper presented at: 2009 IEEE Nuclear Science Symposium Conference Record (NSS/MIC); 2009 Oct 24-Nov 1; Orlando, USA.

85. Degenhardt C, Rodrigues P, Trindade A, Zwaans B, Mülhens O, Dorscheid R, et al. Performance evaluation of a prototype Positron Emission Tomography scanner using Digital Photon Counters (DPC). Paper presented at: 2012 IEEE Nuclear Science Symposium and Medical Imaging Conference Record (NSS/MIC); 2012 Oct 27-Nov 3; Anaheim, USA.

86. Zhang J, Binzel K, Bardos P, Nagar V, Knopp M, Zhang B, et al. FDG dose reduction potential of a next generation digital detector PET/CT system: initial clinical demonstration in wholebody imaging. J Nucl Med. 2015;56 (Suppl 3):1823.

87. Narayanan M, Andreyev A, Bai C, Miller M, Hu Z. TOFbenefits on the philips digital PET/CT scanner: evaluation of faster convergence and reduced scan times. J Nucl Med. 2016;57(Suppl 2):201.

88. Ahn BC. Personalized medicine based on theranostic radioiodine molecular imaging for differentiated thyroid cancer. Biomed Res Int. 2016;2016:1680464.

89. Yordanova A, Eppard E, Kürpig S, Bundschuh RA, Schönberger S, Gonzalez-Carmona M, et al. Theranostics in nuclear medicine practice. Onco Targets Ther. 2017;10:48214828.

90. Choudhury P, Gupta M. Personalized \& precision medicine in cancer: a theranostic approach. Curr Radiopharm. 2017;10:166-170.

91. Koh JM, Kim ES, Ryu JS, Hong SJ, Kim WB, Shong YK. Effects of therapeutic doses of 131I in thyroid papillary carcinoma patients with elevated thyroglobulin level and negative 131I whole-body scan: comparative study. Clin Endocrinol (Oxf). 2003;58:421-427.

92. Jun S, Lee JJ, Park SH, Kim TY, Kim WB, Shong YK, et al. Prediction of treatment response to ${ }^{131} \mathrm{I}$ therapy by diffuse hepatic uptake intensity on post-therapy whole-body scan in patients with distant metastases of differentiated thyroid cancer. Ann Nucl Med. 2015;29:603-612.

93. Lee N, Oh I, Chae SY, Jin S, Oh SJ, Lee SJ, et al. Radiation dosimetry of $\left[{ }^{18} \mathrm{~F}\right] \mathrm{GP} 1$ for imaging activated glycoprotein $\mathrm{IIb} / \mathrm{III}$ receptors with positron emission tomography in patients with acute thromboembolism. Nucl Med Biol. 2019;72-73:45-48.

94. Willowson KP, Eslick E, Ryu H, Poon A, Bernard EJ, Bailey DL. Feasibility and accuracy of single time point imaging for renal dosimetry following ${ }^{177}$ Lu-DOTATATE ('Lutate') therapy. EJNMMI Phys. 2018;5:33.

95. Visvikis D, Cheze Le Rest C, Jaouen V, Hatt M. Artificial intelligence, machine (deep) learning and radio(geno) mics: definitions and nuclear medicine imaging applications. Eur J Nucl Med Mol Imaging. 2019;46:2630-2637.

96. Uribe CF, Mathotaarachchi S, Gaudet V, Smith KC, RosaNeto P, Bénard F, et al. Machine learning in nuclear medicine: part 1-introduction. J Nucl Med. 2019;60:451-458.

97. Goodfellow I, Bengio Y, Courville A. Deep learning. Cambridge: MIT Press; 2016.

98. Choi H. Deep learning in nuclear medicine and molecular imaging: current perspectives and future directions. Nucl Med Mol Imaging. 2018;52:109-118.

99. Weber GM, Mandl KD, Kohane IS. Finding the missing link for big biomedical data. JAMA. 2014;311:2479-2480.

100. Chicklore S, Goh V, Siddique M, Roy A, Marsden PK, Cook GJ. Quantifying tumour heterogeneity in 18F-FDG PET/ CT imaging by texture analysis. Eur J Nucl Med Mol Imaging. 2013;40:133-140.

101. Cook GJ, Yip C, Siddique M, Goh V, Chicklore S, Roy A, et al. Are pretreatment 18F-FDG PET tumor textural features in non-small cell lung cancer associated with response and survival after chemoradiotherapy? J Nucl Med. 2013; 54:19-26.

102. Ganeshan B, Goh V, Mandeville HC, Ng QS, Hoskin PJ, Miles KA. Non-small cell lung cancer: histopathologic correlates for texture parameters at CT. Radiology. 2013; 266:326-336.

103. Ha S, Choi H, Cheon GJ, Kang KW, Chung JK, Kim EE, et al. Autoclustering of non-small cell lung carcinoma subtypes on (18)F-FDG PET using texture analysis: a preliminary result. Nucl Med Mol Imaging. 2014;48:278-286.

104. Lee HS, Oh JS, Park YS, Jang SJ, Choi IS, Ryu JS. Differentiating the grades of thymic epithelial tumor malignancy using textural features of intratumoral heterogeneity via (18)F-FDG PET/CT. Ann Nucl Med. 2016;30:309-319. 
105. Aerts H. Radiomics: there is more than meets the eye in medical imaging. Proc SPIE. 2016:9785:2016SPIE.9785E..0OA.

106. Hatt M, Tixier F, Visvikis D, Cheze Le Rest C. Radiomics in PET/CT: more than meets the eye? J Nucl Med. 2017;58: 365-366.

107. Choi H, Jin KH; Alzheimer's disease neuroimaging initiative. Predicting cognitive decline with deep learning of brain metabolism and amyloid imaging. Behav Brain Res. 2018;344:103-109.

108. Choi H, Kim YK, Yoon EJ, Lee JY, Lee DS; Alzheimer's Disease Neuroimaging Initiative. Cognitive signature of brain FDG PET based on deep learning: domain transfer from Alzheimer's disease to Parkinson's disease. Eur J Nucl Med Mol Imaging. 2020;47:403-412.

109. Choi H, Ha S, Im HJ, Paek SH, Lee DS. Refining diagnosis of Parkinson's disease with deep learning-based interpretation of dopamine transporter imaging. Neuroimage Clin. 2017;16:586-594.

110. Ryoo HG, Choi H, Lee DS. Deep learning-based interpretation of basal/acetazolamide brain perfusion SPECT leveraging unstructured reading reports. Eur J Nucl Med Mol Imaging. 2020;47:2186-2196.

111. Huang B, Chen Z, Wu PM, Ye Y, Feng ST, Wong CO, et al. Fully automated delineation of gross tumor volume for head and neck cancer on PET-CT using deep learning: a dual-center study. Contrast Media Mol Imaging. 2018; 2018:8923028.

112. Chen L, Shen C, Zhou Z, Maquilan G, Albuquerque K, Folkert MR, et al. Automatic PET cervical tumor segmentation by combining deep learning and anatomic prior. Phys Med Biol. 2019;64:085019.

113. Lindgren Belal S, Sadik M, Kaboteh R, Enqvist O, Ulén J, Poulsen $\mathrm{MH}$, et al. Deep learning for segmentation of 49 selected bones in CT scans: first step in automated PET/
CT-based 3D quantification of skeletal metastases. Eur J Radiol. 2019;113:89-95.

114. Park J, Bae S, Seo S, Park S, Bang JI, Han JH, et al. Measurement of glomerular filtration rate using quantitative SPECT/CT and deep-learning-based kidney segmentation. Sci Rep. 2019;9:4223.

115. Wang Y, Yu B, Wang L, Zu C, Lalush DS, Lin W, et al. 3D conditional generative adversarial networks for highquality PET image estimation at low dose. Neuroimage. 2018;174:550-562.

116. Ouyang J, Chen KT, Gong E, Pauly J, Zaharchuk G. Ultralow-dose PET reconstruction using generative adversarial network with feature matching and task-specific perceptual loss. Med Phys. 2019;46:3555-3564.

117. Song TA, Chowdhury SR, Yang F, Dutta J. PET image super-resolution using generative adversarial networks. Neural Netw. 2020;125:83-91.

118. Choi H, Lee DS; Alzheimer's Disease Neuroimaging Initiative. Generation of structural MR images from amyloid PET: application to MR-less quantification. J Nucl Med. 2018;59:1111-1117.

119. Hwang D, Kim KY, Kang SK, Seo S, Paeng JC, Lee DS, et al. Improving the accuracy of simultaneously reconstructed activity and attenuation maps using deep learning. J Nucl Med. 2018;59:1624-1629.

120. Hwang D, Kang SK, Kim KY, Seo S, Paeng JC, Lee DS, et al. Generation of PET attenuation map for whole-body timeof-flight 18F-FDG PET/MRI using a deep neural network trained with simultaneously reconstructed activity and attenuation maps. J Nucl Med. 2019;60:1183-1189.

121. Gong K, Yang J, Kim K, El Fakhri G, Seo Y, Li Q. Attenuation correction for brain PET imaging using deep neural network based on Dixon and ZTE MR images. Phys Med Biol. 2018;63:125011. 